\title{
Neural Networks-based Process Model and its Integration with Conventional Drum Level PID Control in a Steam Boiler Plant
}

\author{
Douglas T. Mugweni \\ Engineering Institute of Technology, Perth, WA 6005, Australia \\ E-mail: 1598053@student.eit.edu.au; \\ Hadi Harb \\ Engineering Institute of Technology, Perth, WA 6005, Australia \\ E-mail: hadi.harb@eit.edu.au;
}

Received: 08 August 2021; Accepted: 01 September 2021; Published: 08 October 2021

\begin{abstract}
Controlling drum level is a major and crucial control objective in thermal power plant steam boilers. The drum level as a controlled variable is highly characterized by complex non-linear process dynamics as well as measurement noise and long-time delays. Developing a data-driven process model is particularly advantageous as it could be built from ongoing operational data. Such a model could be used to assist existing controllers by providing predictions regarding the drum level. The aim of this paper is to develop such a model and to propose a control architecture that can be easily integrated into existing control hardware. For that purpose, different ne ural networks are used, Multilayer Perceptron (MLP), Nonlinear Autoregressive Exogenous (NARX), and Long Short Term (LSTM) neural networks. LSTM and MLP were able to capture the dynamics of the process, but LSTM showed superior performance. The results demonstrate that the use of traditional machine learning criteria to evaluate a process model is not necessarily adequate. Using the model in an open-loop and a closed-loop simulation is more suitable to test its ability to capture the dynamics of the process. A novel architecture that integrates the process model within an existing closed-loop controller is proposed. The architecture uses adaptive weights to ensure that a good model is given more influence than a bad model on the controller's output.
\end{abstract}

Index Terms: Drum level control, Steam boiler plant, Neural Networks, Proportional Integral Derivative control, System identification, Predictive control.

\section{Introduction}

The steam boiler plant is a very critical plant in all industries involved in the production of steam for use in a variety of industrial applications [12,13,14, 15, 16, 17, 18]. In a coal fired power plant, the steam boiler is basically a group of energy conversion equipment that converts chemical energy from fossil fuels like coal, fuel oil or gas into steam energy which will then be used as a source of mechanical energy for driving the turbines that turn the generators. Its primary function is for producing steam for use in electric power generation and for the maintenance of energy locked up in steam in balance with the load demand while retaining the internal variables such as drum level and pressure within optimum and safe operating range as per the plant design [14].

In thermal power plant steam boilers producing steam to drive turbines for electric power generation, the major control objectives are;

(i) To control the generated power output from the generators as required by the power system to which the generators are connected,

(ii) To control the total fuel supply to the boilers for producing the required power,

(iii) To control the boiler drum level and drum pressure,

(iv) To control the final superheated steam temperature and pressure at the outlet of the boiler.

The level of water inside the drum of a power plant steam boiler is arguably the most critical and sensitive variable to control through careful manipulation of the feedwater regulation valves and is a key feature involved in the continuous production of the desired amount of steam as well as complying with the safety and regulatory requirements 
for the safe operation of the plant.

A continuous decline in water level inside the boiler drum will result in the boiler tubes being deprived of their cooling medium and expose them to high thermal energy which then results in tube failures. On the contrary, a continuous increase in drum level will tend to interfere with the process of water and steam separation in the drum, thereby causing a reduction in boiler efficiency and an undesirable effect of water carryover into the steam piping and eventually to the turbine. Therefore, precision in controlling the water level inside the drum of a steam boiler is crucial for the efficient operation of the boiler drum. However, power plant boilers are frequently subjected to changing loads and are more vulnerable to high frequencies of load disturbances. On the other hand, the controlled variable is highly characterized by long process delay, fluctuations in water level and nonlinearity in the drum vessel, as well as process measurement noise.

Controlling the level of water inside a steam boiler drum is quite a daunting task due to inherent process disturbances as well as process dead time. First and foremost, the combustion process itself inside the boiler furnace is a non-linear process that is extremely influenced by the quality of the coal supplied to the boiler for burning in order to produce steam. Secondly, the combined effect of shrinking and swelling of phase behavior due to the integrating nature of drum level dynamics causes fluctuations in water level in the boiler drum. Thirdly, the control strategy implemented as well as the hardware employed to perform this task, i.e. the boiler design, water and steam piping design, the instrumentation design and calibration, final control elements (valves), etc., also have their limitations in achieving effective control of the water level in the boiler drum [22].

One commonly employed strategy for drum level control is shown in Fig.1. below. It utilizes a feedback and feedforward control technique. The feedback controller is a Proportional Integral Derivative (PID) controller and the feedforward controller is a rule-based controller. The main advantage of this strategy is that it aims to compensate for load variations in steam flow out of the boiler and feed water flow demand to the boiler.

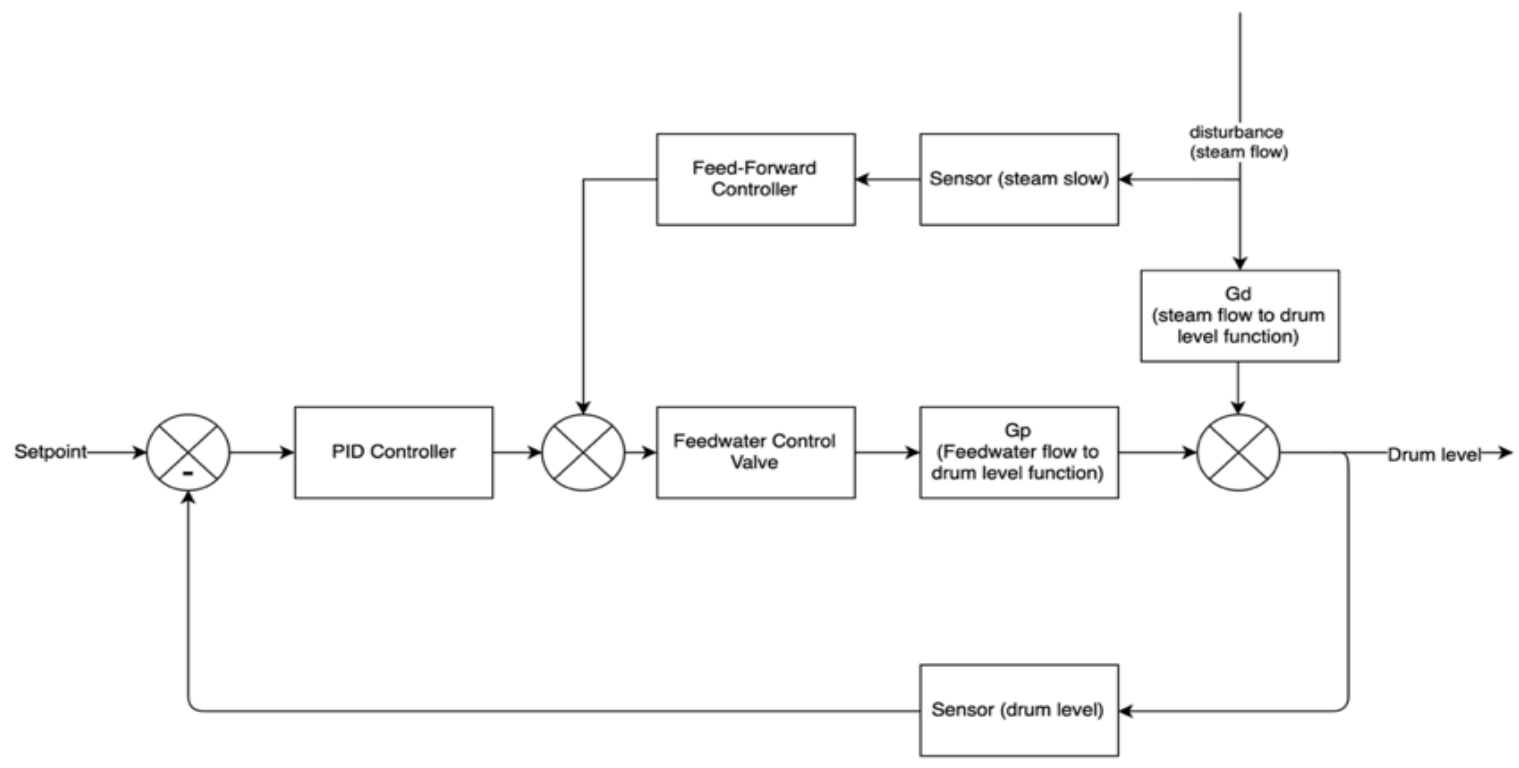

Fig.1. Control strategy.

The controllers should produce suitable control signals based on the given measurements, steam flow and current drum level. To achieve this, a mathematical model of the process should be established first. A model is a simplified mathematical representation of a system or process. In the case of drum level control, the model should describe the relationship between the inputs, steam flow and feedwater flow, and the output, the drum level. A suitable model can be used to make future predictions of the outputs given the inputs. Such predictions, if integrated in the control algorithm, allow for better control. The research objective is hence to obtain a suitable model that can be used to make future predictions of the drum level given the steam flow rate and feedwater flow rate. There are mainly two approaches to obtain a model: first principle and data driven.

Obtaining a model by applying mass and energy balance equations, first principle, tends to produce a second order model that fairly mirrors the dynamics of level and pressure inside the drum [21]. However, a drawback in this model lies in its failure to accurately model the mass of water in the drum. Although the model gives a fairly accurate total mass of water in the system, it fails to account for the steam bubbles inside the risers and below the water surface level inside the drum. Moreover, one needs a detailed knowledge of the process and the equipment to build such models. This is not always feasible. The data-driven alternative to obtaining a model of is by applying machine learning algorithms to predict the output from the inputs[21]. The main challenge in this case is to produce a model that can capture the dynamics of the process. Moreover, we argue that evaluating a machine learning model using traditional evaluation 
criteria such as correlation between the models output and the true output is not necessarily suitable in process modeling. The ability of the model to generalize and to capture the dynamics of the process should be carefully evaluated.

This paper aims at developing a data-driven model that can be trained on existing plant data. The model should capture the dynamics of the process and should be easily implementable within existing control systems.

We compare different Artificial Neural Networks architectures, and we propose an evaluation strategy for the selection of a suitable model. Then, we suggest an architecture that is easily implementable within existing systems.

\section{Related Work}

Several methods for controlling drum level in power plant drum boilers are proposed in the literature ranging from those employing single-input single-output (SISO) PID and fuzzy adaptive techniques [12], to those based on neural networks (NN) [20], and model predictive control (MPC) [19].

In [1] different neural networks architectures were evaluated on the task of predicting heat rate and boiler efficiency from actual plant data. The paper is not specifically dealing with water level prediction in steam boilers, but it provides a comparison of machine learning models that are trained on a real plant data. Neural networks were investigated in [2] as models for the prediction of fresh steam properties of a coal-fired boiler using real plant data. In this paper also, the relevance to our work is the use of real plant data even though the main objective is not drum level prediction.

Steam properties predictions using neural networks was also investigated in[3] using real plant data. However, the time resolution of the data is 30 minutes and the authors did a manual outlier elimination.

More related to our work is [21] where the authors used Nonlinear Autoregressive Exogenous Neural Network (NARX NN) architecture to predict drum level and pressure from inputs that are feedwater flow, steam flow and heat input. The data was obtained from an open loop simulation of a first principle model. The model obtained was evaluated on the open loop data and on step change in the inputs. The model showed good results. However, the evaluation and the development of the model were carried out on simulated open loop data, which is a major limitation when one has to apply such a model in the control of a real plant.

The authors in [20] also carried out similar work and went on to propose a methodology based on what is called heuristic technique for developing system identification models through application of NARX NN to predict the drum level in a coal fired power plant also in India. The drawbacks in these works were the resolution of the data used for training the neural networks, recorded on hourly basis, which would severely affect the accuracy of the drum level predictions and in turn would also affect the precision in the control of the level. The second drawback is the lack of a sufficiently accurate methodology for the safe implementation and integration of the proposed control techniques into an existing plant.

Besides [20] and [21] where an attempt was made to explore the possible application of neural networks to drum level prediction and control in coal fired power plants, there has not been much reference in the literature to the practical integration of data-driven modeling techniques with traditional PID control of drum level on existing thermal power plant steam boilers. This paper attempts to fill this gap by proposing opportunities for the selection of machine learning models in controlling the water level in a power plant drum boiler and their integration with the conventional PID controller in cascade control configuration.

\section{Neural Networks-based Process Modelling}

Artificial Neural Networks are powerful machine learning tools having the capability to identify highly complex relationships given input and output data only. An Artificial Neural Network (ANN) is composed of neurons that are connected and organized in layers. Each neuron implements a relatively simple operation of summing its inputs and applying a function on the result. The output of a neuron is then sent as inputs to other neurons. Neurons are organized in layers, input, hidden, and output layers. The ANN maps an input vector to an output vector as shown in Fig.2. below. Each connection between two neurons has a weight that is called synaptic weight. The process of training an ANN is mainly the process of finding the synaptic weights that will allow it to produce the desired outputs for given inputs [24].

ANNs are commonly applied to model a process from input and output data. They are becoming an interesting feature in control systems since they can now be easily implemented and integrated into existing hardware such as in Programmable Logic Controllers (PLCs) and in Distributed Control Systems (DCSs) [11].

In a process modelling scenario, the ANN's task is to predict the future value of the output given current and past values of it along with current and past values of input variables. In such scenarios, the inputs typically correspond to manipulated and disturbance variables and the output to the controlled variable. In the drum level prediction application, the output is the drum level and the possible inputs are feedwater flow, steam flow, heat input, and drum pressure. In this work we concentrate on the two central inputs that are feedwater and steam flow. From a mass balance perspective, these variables could explain the drum level variations although with delays. 


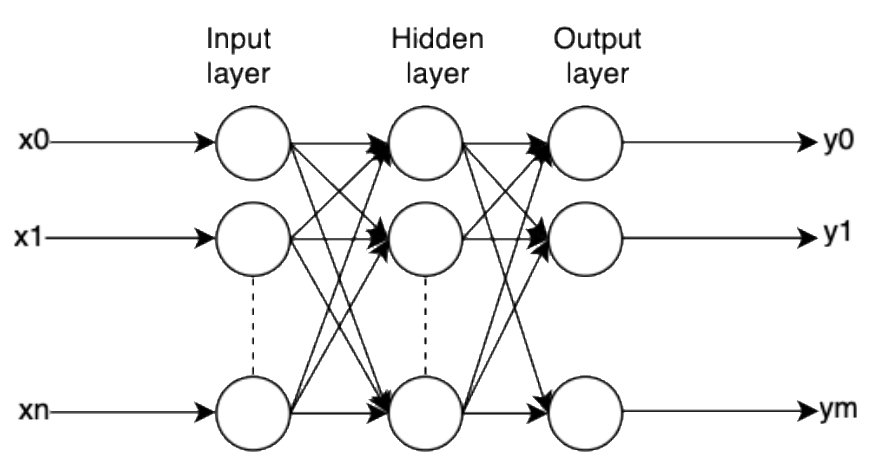

Fig.2. An Artificial Neural Network architecture.

\subsection{The data}

Data was collected from one of the boiler plants at Camden Power Station, a coal fired power plant situated in the Mpumalanga Province of South Africa, owned and operated by the Generation Division of Eskom Holdings SOC. The data collected was captured in the plant historian of the automation system on 22-08-2019 between the period of 03:00 and 06:05. In total 9300 data samples were collected at 1 second resolution. Heat input, feedwater flow, steam flow, drum pressure, and drum level measurements were obtained. As mentioned above, we concentrated on feedwater flow, steam flow, and drum level where the objective is to predict a future drum level from current and previous feedwater flow, steam flow, and drum level measurements. It is worth noting that the data was collected from a running system in a closed-loop fashion. Hence, feedwater flow is manipulated by a controller that uses drum level measurement and steam flow to calculate the control signal. Consequently, feedwater flow should correlate with steam flow. For example, if steam demand increases, the steam flow increases leading to a corrective action from the feed-forward controller. In parallel, this leads to a delayed decrease in drum level that will cause the feedback controller to generate a correction action as well.

The data was not filtered or manually selected, and hence it contains any possible noisy measurements provided by the sensors.

Fig.3. below shows the steam flow and feedwater flow as a function of time. The unit of both is in $\mathrm{kg} / \mathrm{s}$. The dotted lines correspond to the moving averages of the curves. By analyzing the curves one can notice that the feedwater signal has a similar shape to that of the steam flow but with a delay. An increase in steam demand results in a delayed increase of feedwater flow. The delay is approximately 150 seconds.

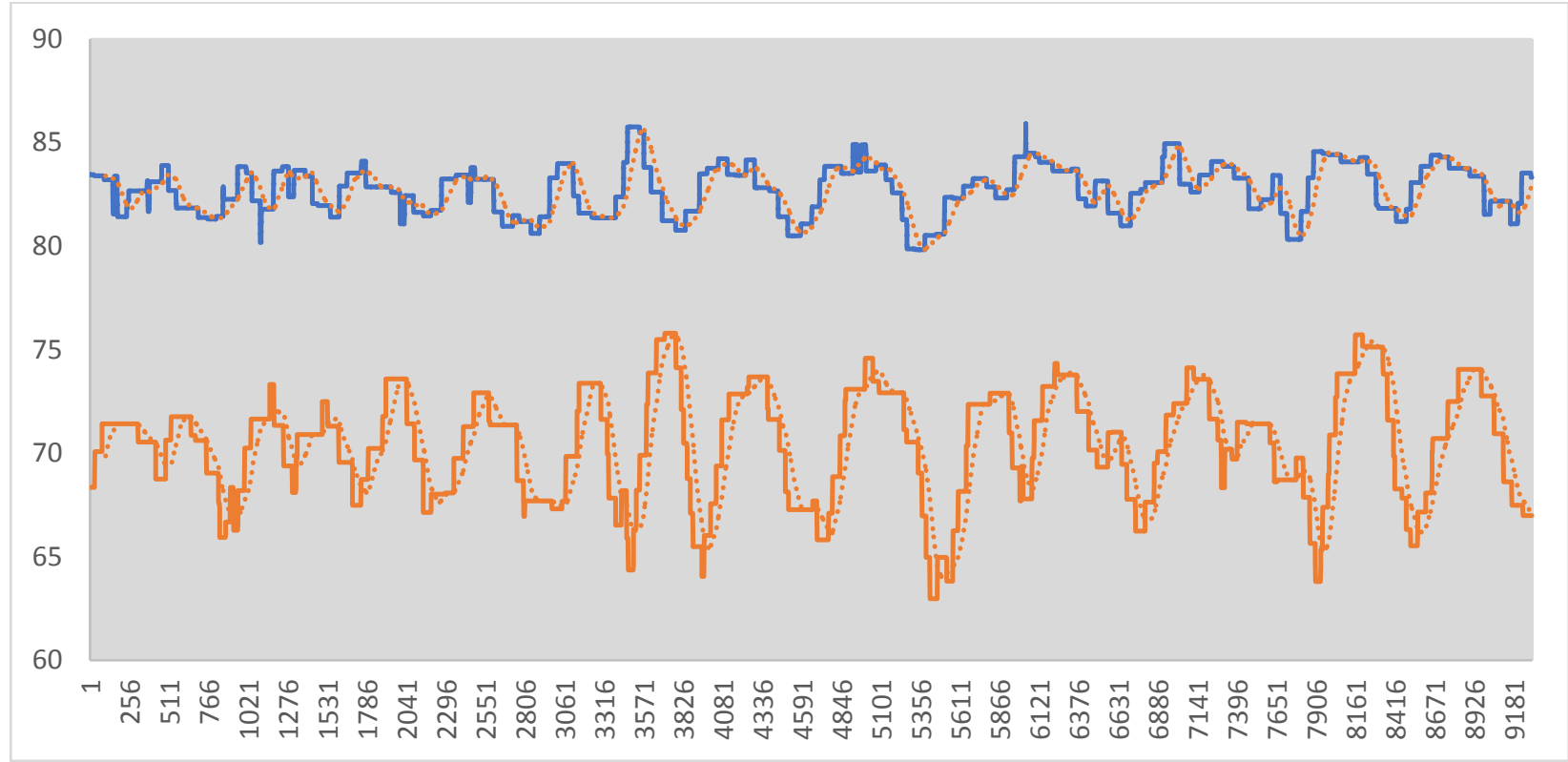

Fig.3. Steam flow (upper) and feedwater (bottom) curves. The phase shift could be seen where an increase in steam demand results in a delayed increase of feedwater $(\approx 150$ s delay). 


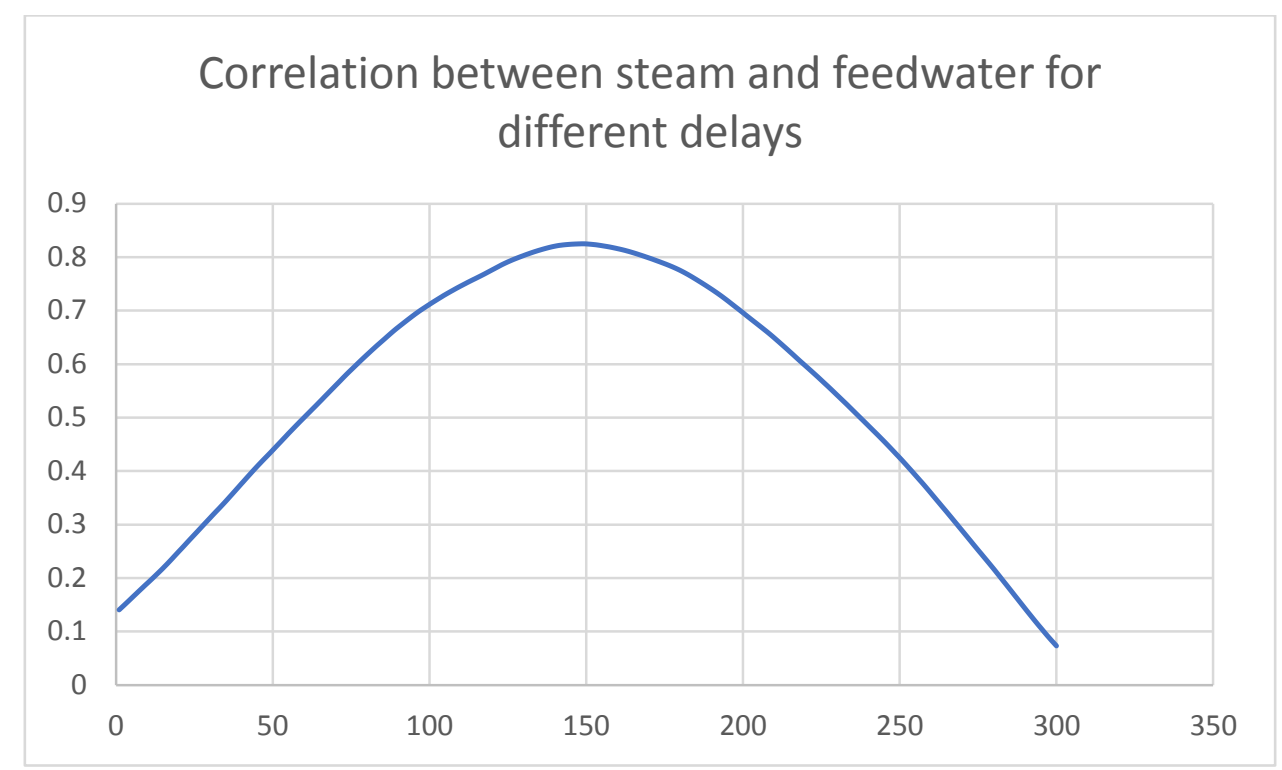

Fig.4. Correlation between Steam and Feedwater delayed by t time. The $\mathrm{x}$-axis represents the delay and the $\mathrm{y}$-axis the correlation coefficient $\mathrm{R}$.

The correlation between steam flow and feedwater flow is shown as a function of time delay in Fig.4. One can notice that the correlation is at its maximum at around 150 seconds delay. It is hence expected that the process has comparable delays.

\subsection{Features}

The input features are feedwater flow and steam flow. The drum level is the output variable. However, since all variables are time-domain signals, one could benefit from using current and previous output values along previous input values. Since mass balance is essentially what dictates the drum level [4], we considered also the difference between steam flow and feedwater flow as an alternative input feature.

\subsection{Neural Network architecture}

Different Neural Network architectures could be used. In this work we considered three architectures: Multilayer Perceptron (MLP), Nonlinear autoregressive exogenous model (NARX), and Long-Short Term Memory (LSTM).

MLP architecture is a Feedforward Neural Network architecture that is commonly applied in machine learning and system identification [6]. In the architecture that we selected, the MLP has to predict the future drum level from the current features and drum level. No sequence information such as previous feature values or drum levels was included. After initially trying different architectures we used an MLP with one hidden layer containing 50 hidden neurons. The MLP was used to select the features that will be subsequently used in other architectures.

Formally the problem is to train an MLP that represent the functions g and g' below:

$$
\begin{gathered}
L(t+i)=g(S(t), F(t), L(t)) \\
L(t+i)=g^{\prime}(\Delta(t), L(t))
\end{gathered}
$$

Where,

$\mathrm{S}(\mathrm{t})$ is the steam flow at time $\mathrm{t}$,

$\mathrm{F}(\mathrm{t})$ is the feedwater flow at time $\mathrm{t}$,

$\mathrm{L}(\mathrm{t})$ is the drum level at time $\mathrm{t}$,

$\Delta(\mathrm{t})$ is steam flow minus feedwater flow at time $\mathrm{t}$.

$\mathrm{i}$ is a constant,

$\mathrm{g}$ is the prediction function when using $\mathrm{S}, \mathrm{F}$, and $\mathrm{L}$ as features,

$\mathrm{g}$ ' is the prediction function when using $\Delta$, and $\mathrm{L}$ as features.

Fig.5. below shows the MLP architecture used to represent g'. 


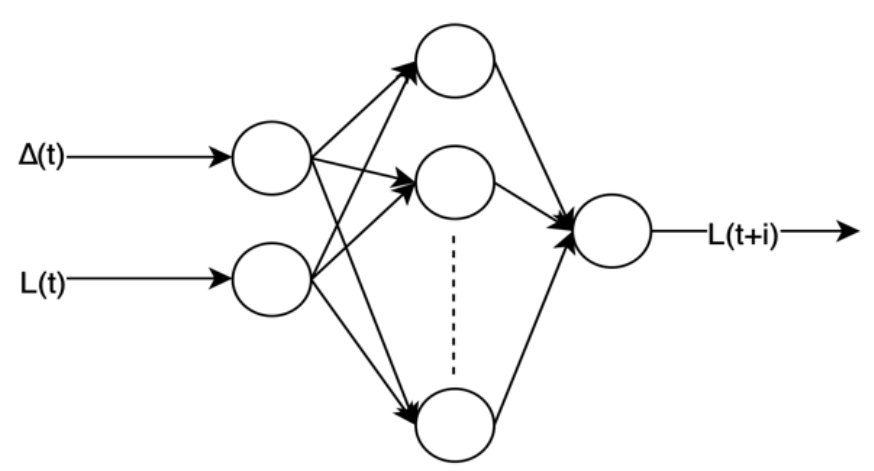

Fig.5. MLP architecture used. $\Delta$ corresponds to Steam - Feedwater. and L to level.

\subsubsection{NARX}

Nonlinear autoregressive exogenous model (NARX) could be used to model the process [5]. One of the advantages of NARX is that it takes into account features and outputs at different time steps to predict future output.

Formally the problem is to train a NARX that represents the functions g" Fig.6.

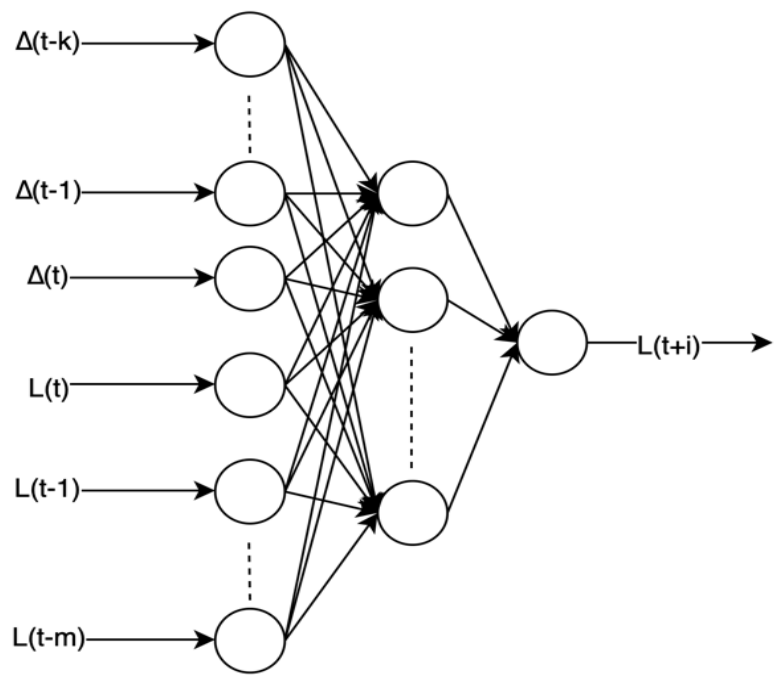

Fig.6. Architecture used for NARX.

$$
L(t+i)=g^{\prime \prime}(\Delta(t), \Delta(t-1), \ldots, \Delta(t-k), L(t), L(t-1), \ldots, L(t-m))
$$

Where,

$\mathrm{L}(\mathrm{t})$ is the drum level at time $\mathrm{t}$,

$\Delta(\mathrm{t})$ is steam flow minus feedwater flow at time $\mathrm{t}$.

$\mathrm{i}, \mathrm{k}$ and $\mathrm{m}$ are constants,

$\mathrm{g}^{\prime \prime}$ is the prediction function.

\subsubsection{LSTM}

Long-Short Term Memory (LSTM) models are a type of Recurrent Neural Networks (RNN) that gained popularity due to their ability to model sequences $[7,8,9]$. One can use LSTM to model a time sequence and consequently predict future values from previous ones. Fig.7. below shows an LSTM architecture as applied to the process modeling problem.

The feedback connection shown in the hidden layer represents the LSTM units where the current output of a neuron is a function of its current input and its previous state (previous neuron output). 


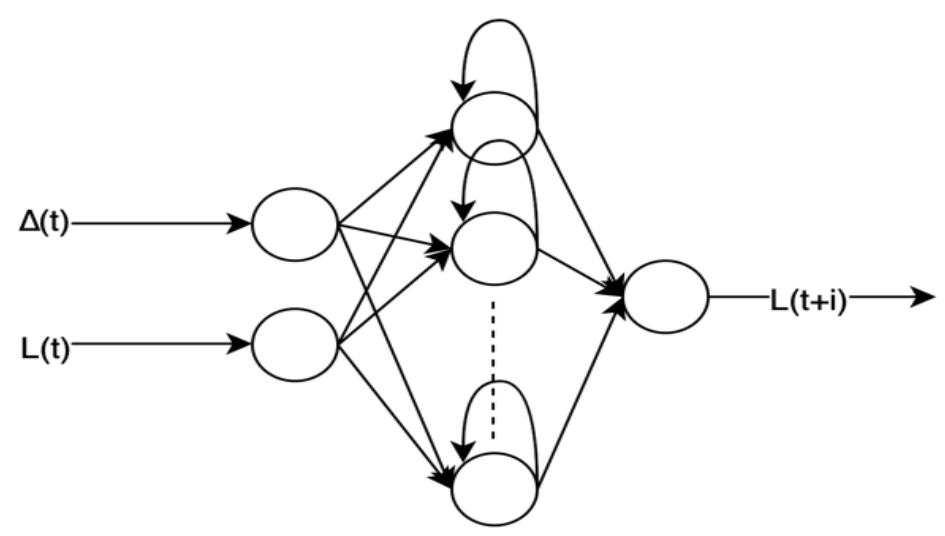

Fig.7. LSTM architecture used.

Each of the architectures presented above, MLP, NARX, and LSTM, has its advantages and disadvantages. MLP is relatively simple with less parameter than NARX and LSTM. NARX encodes the time information in the input, which is interesting as the input signal is a time varying signal. It necessitates however more training data than MLP because it has more parameters to estimate. LSTM also encodes time information, but its training is more complex than that of MLP and NARX.

\section{Simulation}

The collected data was split into $70 \%$ training set, $15 \%$ validation set and $15 \%$ test set. This split is common in machine learning [25]. MATLAB Deep Learning Toolbox [10] was used to train and evaluate the models.

Predictions at different future time steps were evaluated, namely at $1 \mathrm{~s}, 3 \mathrm{~s}, 30 \mathrm{~s}$, and $150 \mathrm{~s}$. We experimented four neural network models, namely:

- MLP with three-dimensional feature vector (steam flow, feedwater flow, drum level)

- MLP with two-dimensional feature vector (steam - feedwater, drum level)

- NARX with two-dimensional feature vector (steam - feedwater, drum level)

- LSTM with two-dimensional feature vector (steam - feedwater, drum level)

The models will be called in the rest of the paper MLP, MLP- $\Delta$, NARX- $\Delta$, and LSTM- $\Delta$ respectively.

The models were evaluated using two different methods. The first is a commonly used method in machine learning and the second is a process control oriented method. In the former Pearson's R correlation coefficient between the model's output and the true values is used. In the latter the model is used to simulate a plant with disturbance injection.

\subsection{Regression evaluation for ( $1 \mathrm{~s}, 3 \mathrm{~s}, 30 \mathrm{~s}, 150 \mathrm{~s})$}

Table 1. Pearson's R correlation coefficient for the different models and different future predictability

\begin{tabular}{|c|c|c|c|c|}
\hline & $1 \mathrm{~s}$ & $3 \mathrm{~s}$ & $30 \mathrm{~s}$ & $150 \mathrm{~s}$ \\
\hline MLP & 0.99 & 0.96 & 0.84 & 0.85 \\
\hline MLP- $\Delta$ & 0.99 & 0.96 & 0.81 & 0.71 \\
\hline NARX- $\Delta$ & 0.97 & 0.94 & 0.83 & 0.74 \\
\hline LSTM- $\Delta$ & 0.98 & 0.95 & 0.71 & 0.67 \\
\hline
\end{tabular}

As one can see in the Table 1 above, all models produce good results up to $150 \mathrm{~s}$ predictability. In particular, the models produce a very high correlation coefficient when predicting the short-term drum level, at $1 \mathrm{~s}$ and $3 \mathrm{~s}$. This is probably due to the fact that drum level does not dramatically change in this time interval. Hence, a model that predicts the next drum level as the same as the current drum level will obtain a good correlation coefficient.

\subsection{Injecting step disturbance on feedwater flow}

The process models were used to predict the drum level for an input signal corresponding to a constant steam flow at $82 \mathrm{~kg} / \mathrm{s}$ and a varying feedwater flow between two constant values, namely $70 \mathrm{~kg} / \mathrm{s}$ and $71 \mathrm{~kg} / \mathrm{s}$. The feedwater signal changed every 200 seconds. The drum level used at time instant $t$ at the input of the model is a previously predicted value from the same model at $t-1$. It is worth noting that this simulation is an open-loop simulation with no controller being employed. 
Due to the nature of the process, it is expected that an increase in feedwater flow results in an increase in drum level. It is important to note that the process is an integrating process; hence a step change in feedwater flow should result in a continuous increase in the drum level [4].
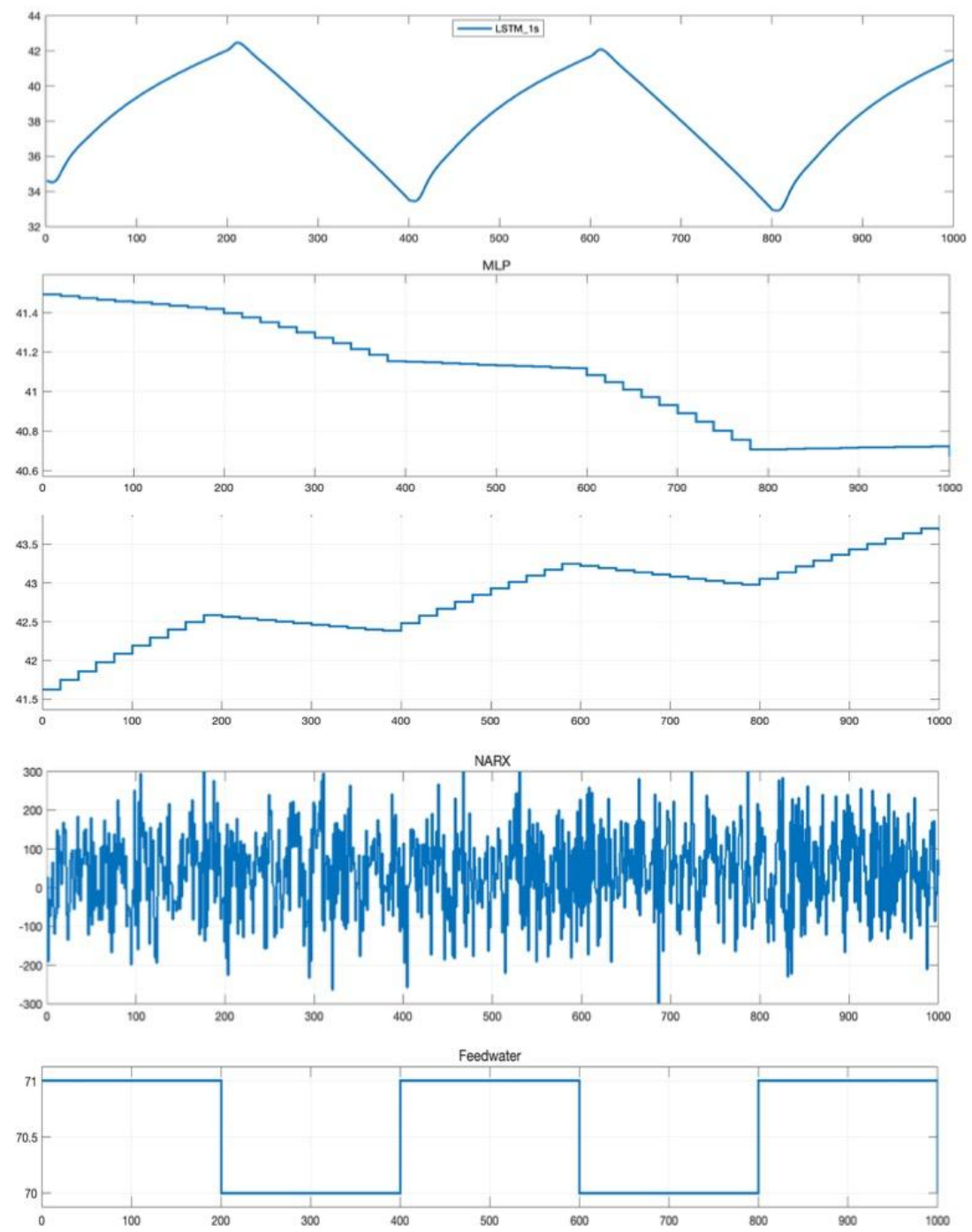

Fig.8. Response of different process models to a change in feedwater.

As can be seen in the Fig.8. above, LSTM- $\Delta$ and MLP- $\Delta$ produced results that are coherent with the expectations. The difference between them is probably due to the input/output stability level. For example, MLP- $\Delta$ is considering feedwater flow at $70 \mathrm{~kg} / \mathrm{s}$ as sufficient to maintain the drum level almost constant. On the contrary, LSTM- $\Delta$ is considering $70 \mathrm{~kg} / \mathrm{s}$ as lower than the necessary flow to maintain a constant drum level. Both show an increase of drum level at $71 \mathrm{~kg} / \mathrm{s}$.

MLP model is not producing acceptable results. In fact, an increase in feedwater results in a decrease in drum level, which is inconsistent with the process.

NARX does not provide satisfactory results. The model's predictions lacked smoothness, i.e. rapidly changing random values was obtained. This is probably due to the nature of the training data used, closed-loop operational data which is not smooth as seen previously. It seems that NARX overfits the training data and captures the short-term changes in drum level instead of the long-term trends. It is worth noting that the short-term changes in drum level could be due to noise in the measurements.

\subsection{Discussion}

The obtained results suggest that a good performance from a machine learning perspective of a model, i.e. using Pearson's correlation coefficient, does not necessarily mean a model is usable in a process control application. On the contrary, LSTM- $\Delta$ that gave the lowest score in correlation evaluation produced the most coherent output when used as a process model. This tends to confirm our hypothesis that the ability of a model to capture the process dynamics cannot 
be evaluated by using the correlation between the model's output and the true output only.

Including more features at the input layer by adding previous time steps such as the case of NARX- $\Delta$ did not improve the process model performance. One explanation is that the model overfits the training data that are not smooth due to their closed-loop real-world nature.

Comparing MLP- $\triangle$ and MLP process model results suggests that using the difference between steam flow and feedwater flow as one input is better than the use of both variables as two independent inputs.

LSTM- $\Delta$ and MLP- $\Delta$ captured the underlying dynamics of the process while NARX- $\Delta$ and MLP did not. However, using MLP- $\Delta$ is more suitable due to the ease of implementation of an MLP on existing industrial hardware such as Programmable Logic Controllers. In the next section we therefore consider MLP- $\Delta$ as the plant model.

\section{Integration}

One use of a process model is for simulation purposes using software like Simulink [10]. The model could be used to tune a PID controller and investigate different control architectures before their integration in a real control system. The MLP- $\Delta$ was used as a process model in a feedback control architecture in a disturbance rejection and a reference tracking scenario. The objective of this simulation is to evaluate the ability of the process model to produce coherent results when used in a closed-loop architecture with a controller in place.

Once a good process model is available, one could benefit from integrating it into an existing control architecture. In fact, the process model could be used to predict inevitable changes in the drum level so that the controller can anticipate such changes by sending corrective signals early on. The integration should be practical however and should not cause any safety concerns.

\subsection{Reference tracking and disturbance rejection}

Fig.9. below shows the control architecture with a PID controller. The controller compares the current drum level obtained from the process model to a setpoint. It then generates a signal to manipulate feedwater flow. Consequently, if steam flow increases, the controller should increase the feedwater flow to keep the drum level at its setpoint. On the other hand, if the setpoint changes, the controller should change the feedwater flow to drive the drum level to the target value.

The PID controller was tuned using trial and error. MLP- $\Delta$ model constitutes the process model. As seen in Fig. 10. and Fig.11. below, the controller is effectively producing coherent control signals. In Fig.9. the feedwater flow follows the steam flow to keep the drum level at its setpoint. In Fig.11. when the setpoint increases, the feedwater flow also increases for about $500 \mathrm{~s}$ then slowly decreases before stabilizing at its original value. This suggests that the MLP- $\Delta$ model is effectively capturing the dynamics of the process by providing coherent results.

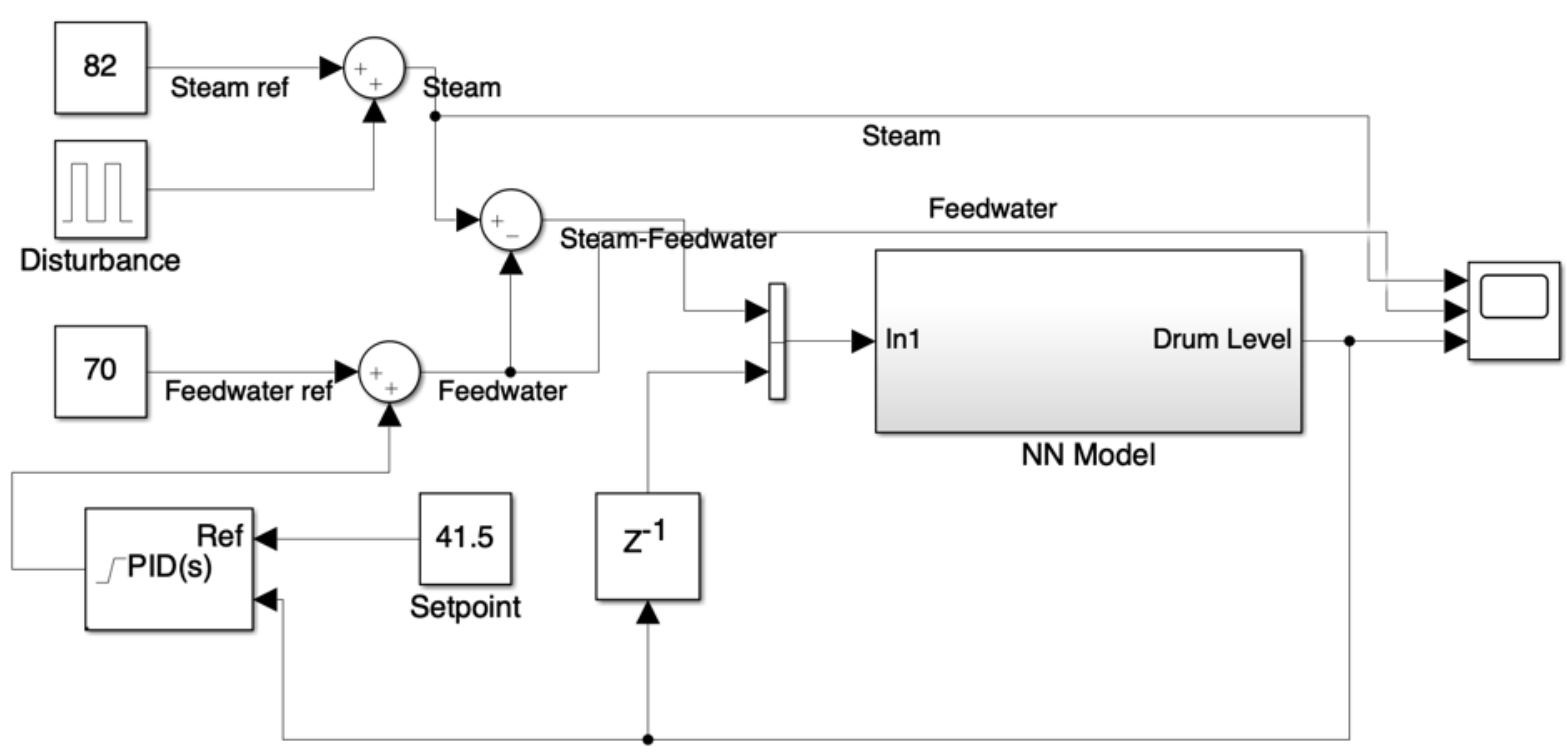

Fig.9. Control architecture using the neural network process model for simulation and tuning purposes. 


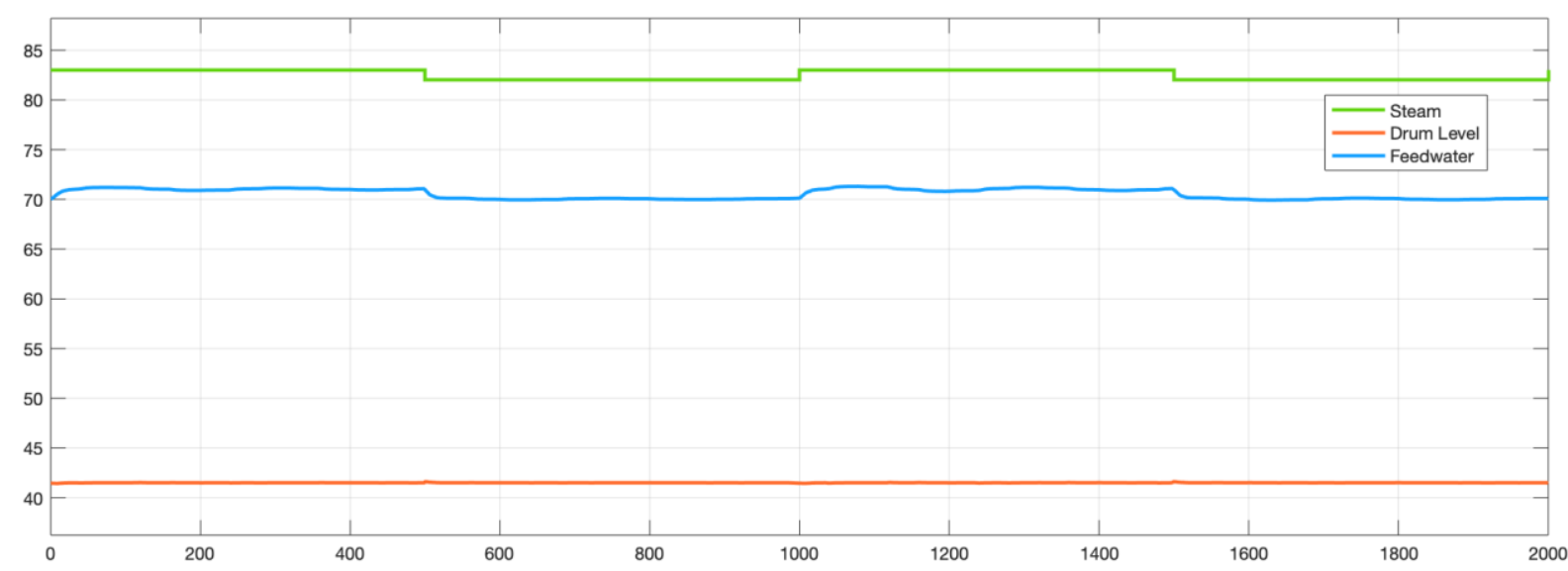

Fig.10. Disturbance rejection results.

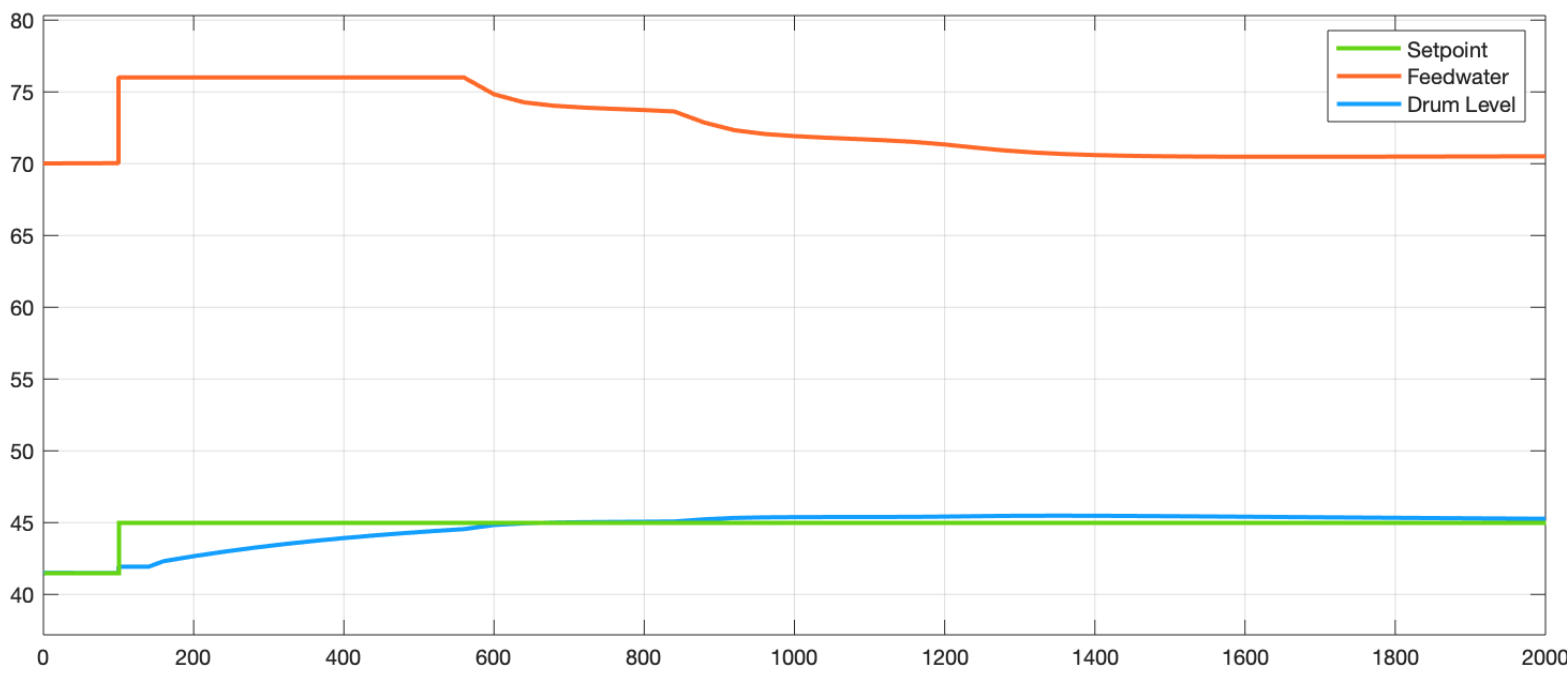

Fig.11. Setpoint tracking results.

\subsection{Integrating predictions into a conventional architecture}

Fig.12. below shows a suggested architecture to integrate the MLP- $\Delta$ process model into an existing control system. The process model predicts the drum level in the future given current steam and feedwater flow, and the previous drum level. The predicted drum level is then combined with the measured one using suitable weights. The weights could be made dynamic such as a high performing model will be given higher weights than a low performing one. The performance of a model could be constantly calculated by calculating the deviation between predicted and measured drum levels. In such configuration, the system will adapt without posing safety and operational concerns. If the model performs well, it will improve the controller's performance by permitting it to anticipate changes. Otherwise, the model weight will be low so that it will not affect the controller's performance.

It is worth noting that the process model could also be LSTM- $\Delta$. However, the ease of implementation of MLP on a Programmable Logic Controller makes it a better choice. 


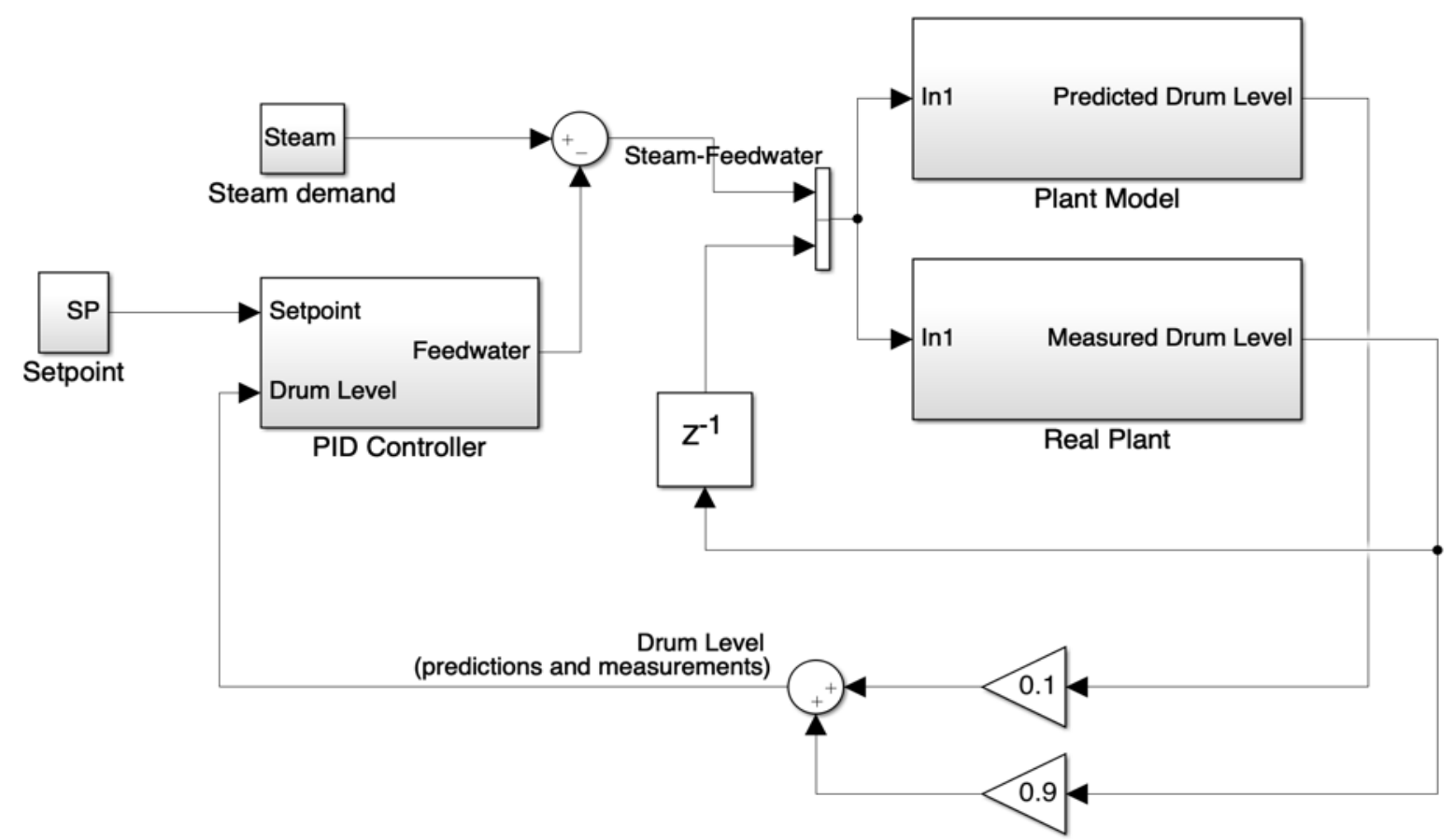

Fig.12. Suggested architecture combining real measurements and predictions. The weights 0.1 and 0.9 are arbitrarily chosen here and could be made dynamic as a function of the prediction quality.

\subsection{Discussion}

The simulation results presented in section 4. showed that both MLP and LSTM could be effectively used as process models that capture its dynamics. MLP is preferred to LSTM because of its ease of implementation on control hardware. When used in a closed-loop architecture employing a PID controller, the MLP process model showed a reasonable behavior for both set point tracking and disturbance rejection. This further confirms the ability of the suggested model to capture the process dynamics by using operational data for training. The suggested architecture presented in Fig.12. achieves the objective of ease of implementation. In fact, the model can generate predictions in parallel to the operation of an existing controller. The controller integrates the predictions by using adaptive weights that reflect the accuracy of the model.

\section{Conclusion}

This paper investigated the use of neural networks as a process model in the context of drum level control in a steam boiler plant. The model is trained on real world closed-loop process data. The neural networks predict the drum level based on current and previous measurements of drum level, steam flow, and feed water flow. We have shown that the use of steam flow minus feed water flow as one feature is more suitable than the use of both variables separately in the feature vector.

We compared three neural networks architectures, namely Multilayer Perceptron (MLP), Nonlinear Autoregressive Exogenous (NARX) neural network, and Long Short Term (LSTM) neural network. The correlation between the model's output and the true drum level measurement was used as an evaluation of the models from a machine learning perspective. We evaluated the models from a process control perspective by using them as plant models in a disturbance injection scenario. Although all neural networks architectures performed well from a machine learning perspective, only LSTM and MLP were able to produce coherent results from a process control perspective. The results suggest that traditional machine learning criteria are not necessarily adequate to evaluate a process model. Moreover, we showed that LSTM produced the most coherent results as process models.

A control architecture that integrates the obtained process model within an existing control system was suggested. The architecture does not pose safety and operational challenges since it uses the process model as a complement to drum level measurements. We propose the use of adaptive weights for the model such as a good model getting higher weights and bad one getting little influence on the controller's output.

MLP is currently a good choice for implementation on a PLC and the control hardware. However, LSTM shows promising results in capturing the dynamics of the process. Consequently, more work needs to be done in the direction of comparing MLP and LSTM as process models integrated in operational control architecture. Furthermore, the adaptive weights idea needs to be evaluated in a real system. 


\section{References}

[1] Deshpande, Purva, Nilima Warke, Prakash Khandare, and Vijay Deshpande. "Thermal power plant analysis using artificial neural network." In 2012 Nirma University International Conference on Engineering (NUiCONE), pp. 1-6. IEEE, 2012.

[2] Smrekar, J., Assadi, M., Fast, M., Kuštrin, I., \& De, S. (2009). Development of artificial neural network model for a coal-fired boiler using real plant data. Energy, 34(2), 144-152.

[3] Smrekar, J., Pandit, D., Fast, M., Assadi, M., \& De, S. (2010). Prediction of power output of a coal-fired power plant by artificial neural network. Neural Computing and Applications, 19(5), 725-740.

[4] ÅSTRÖM, Karl Johan et BELL, Rodney D. Drum-boiler dynamics. Automatica, 2000, vol. 36, no 3, p. 363-378.

[5] CHEN, Sheng, BILLINGS, S. A., et GRANT, P. M. Non-linear system identification using neural networks. International journal of control, 1990, vol. 51, no 6, p. 1191-1214.

[6] Kumpati, S. Narendra, and Parthasarathy Kannan. "Identification and control of dynamical systems using neural networks." IEEE Transactions on neural networks 1.1 (1990): 4-27.

[7] Duan, Yanjie, Yisheng Lv, and Fei-Yue Wang. "Travel time prediction with LSTM neural network." 2016 IEEE 19th International Conference on Intelligent Transportation Systems (ITSC). IEEE, 2016.

[8] Sagheer, Alaa, and Mostafa Kotb. "Time series forecasting of petroleum production using deep LSTM recurrent networks." Neurocomputing 323 (2019): 203-213.

[9] Zhao, Zheng, et al. "LSTM network: a deep learning approach for short-term traffic forecast." IET Intelligent Transport Systems 11.2 (2017): 68-75.

[10] MATLAB 2019b, the MathWorks, Inc., Natick, Massachusetts, United States.

[11] Wedajo T. Abdisa, Hadi Harb, "A Neural Network Based Motor Bearing Fault Diagnosis Algorithm and its Implementation on Programmable Logic Controller", International Journal of Intelligent Systems and Applications (IJISA), Vol.11, No.10, pp.1-14, 2019.

[12] HU, L. J., ZHANG, Ke, et LIU, Tao. Study on the boiler drum water level based on fuzzy adaptive control. In: 24th Chinese Control and Decision Conference (CCDC). 2012. p. 1659-1663.

[13] LU, C. X., REES, N. W., et DONALDSON, S. C. The use of the Åström-Bell model for the design of drum level controllers in power plant boilers. IFAC Proceedings Volumes, 2005, vol. 38, no 1, p. 139-144.

[14] Sunil, P. U., Jayesh J. Barve, and PS V. Nataraj. "Boiler drum-level control using QFT." 2013 Nirma University International Conference on Engineering (NUiCONE). IEEE, 2013.

[15] Qiliang, Yang, Xing Jianchun, and Wang Ping. "Water level control of boiler drums using one IEC61131-3-based DCS." 2007 Chinese Control Conference. IEEE, 2007.

[16] Gowthaman, E., et al. "Performance analysis of hybrid fuzzy-PID controller action on boiler drum level control." 2016 Online International Conference on Green Engineering and Technologies (IC-GET). IEEE, 2016.

[17] A. Kozáková, S. Bucz "Multiloop control of a drum boiler", Journal of Electrical Systems and Information Technology 1 (2014) $26-35$.

[18] Boesack, Craig D., Priyanka Thakur, and Albert Smit. "Experiences on power plant modelling and its parameter estimation.” International Journal of Intelligent Information Processing 5.3 (2015): 67.

[19] R. Horalek, J. Hlava, "Comparison of Linear and Nonlinear Model Predictive Control of Benchmark Drum Boiler", Annals of DAAAM for 2011 \& Proceedings of the 22nd International DAAAM Symposium, Volume 22, No. 1, ISSN 1726-9679.

[20] Selvi, BS Thamarai, D. Kalpana, and T. Thyagarajan. "Modeling and prediction of boiler drum in a thermal power plant." 2017 Trends in Industrial Measurement and Automation (TIMA). IEEE, 2017.

[21] Oko, Eni, Meihong Wang, and Jie Zhang. "Neural network approach for predicting drum pressure and level in coal-fired subcritical power plant." Fuel 151 (2015): 139-145.

[22] G. A. Oluwande, "Exploitation of Advanced Control Techniques in Power Generation", Computing and Control Engineering Journal, P63-67, April 2001, Institution of Engineering and Technology (IET).

[23] Horvath, Gábor. "Neural Networks in Systems Identification". Neural Networks for Instrumentation, Measurement and Related Industrial Applications, Edited by S. Ablameyko, L. Goras, M. Gori and V. Piuri. NATO Science Series 185 (2002): 43-78

[24] Haykin, S. Neural Networks, A Comprehensive Foundation, Prentice Hall, New Jersey. 1994

[25] Nguyen Q. et al., "Influence of Data Splitting on Performance of Machine Learning Models in Prediction of Shear Strength of Soil”, Mathematical Problems in Engineering, vol. 2021.

\section{Authors' Profiles}

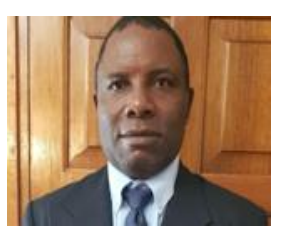

Douglas T. Mugweni. Douglas Mugweni was born in Harare, Zimbabwe on the $4^{\text {th }}$ of September 1974. Douglas obtained his first B.Eng(Honours) degree in electronic engineering from the National University of Science and Technology (NUST), in the city of Bulawayo, Zimbabwe in 1999. Twenty years later in 2020, he obtained his M.Eng in industrial automation from the Engineering Institute of Technology in Perth, Australia.

He joined the Zimbabwe Power Company as a Graduate Trainee Engineer in control and instrumentation and rose to become Senior Engineer. In 2007, he moved to South Africa and joined the power utility Eskom in the Generation Division as a Control and Instrumentation Engineer based at its Camden Power Station plant in Ermelo in the province of Mpumalanga. 
Engineer Mugweni is registered as a professional engineer with the Engineering Council of South Africa (ECSA), a senior member of the South African Institute of Electrical Engineers (SAIEE), and a corporate member of the Institute of Engineering and Technology (IET) in UK.

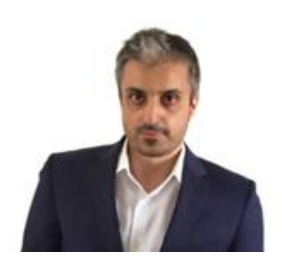

Hadi Harb Hadi Harb holds a MEng (2000) in electrical-electronic engineering from the Lebanese University. He earned his MSc in 2001 and PhD in 2004 both in computer science from the Institut National des Sciences Appliquées INSA Lyon France, and the Ecole Centrale de Lyon France, respectively. In 2004 he joined Centrale Lyon Innovation SA as a research engineer. In 2006 he founded and managed Ghanni, a company specialised in multimedia content recommendation and identification. Several European radio stations and websites licensed Ghanni's music recommendation technology. In 2015 he restructured Ghanni to transform it into a consultancy company in the domain of Artificial Intelligence and joined the Engineering Institute of Technology, Australia, as a lecturer. His current research interests are in the use of Artificial Intelligence techniques to solve industrial problems.

How to cite this paper: Douglas T. Mugweni, Hadi Harb, " Neural Networks-based Process Model and its Integration with Conventional Drum Level PID Control in a Steam Boiler Plant ", International Journal of Engineering and Manufacturing (IJEM), Vol.11, No.5, pp. 1-13, 2021. DOI: 10.5815/ijem.2021.05.01 